AperTO - Archivio Istituzionale Open Access dell'Università di Torino

\title{
Interactive chart of story characters' intentions
}

\section{This is the author's manuscript}

Original Citation:

Availability:

This version is available http://hdl.handle.net/2318/1615308

since 2017-12-04T09:38:37Z

Publisher:

Springer International Publishing

Published version:

DOI:10.1007/978-3-319-48279-8_39

Terms of use:

Open Access

Anyone can freely access the full text of works made available as "Open Access". Works made available under a Creative Commons license can be used according to the terms and conditions of said license. Use of all other works requires consent of the right holder (author or publisher) if not exempted from copyright protection by the applicable law. 
This is the author's final version of the contribution published as:

Lombardo, Vincenzo; Pizzo, Antonio; Damiano, Rossana; Terzulli, Carmi; Albert, Giacomo. Interactive chart of story characters' intentions, in: Interactive Storytelling, 9th International Conference on Interactive Digital Storytelling, ICIDS 2016, Los Angeles, CA, USA, November 15-18, 2016, Proceedings, Springer International Publishing, 2016, 978-3-319-48278-1, pp: 415-418.

The publisher's version is available at:

http://link.springer.com/content/pdf/10.1007/978-3-319-48279-8_39

When citing, please refer to the published version.

Link to this full text:

http://hdl.handle.net/2318/1615308 


\title{
Interactive chart of story characters' intentions
}

\author{
Vincenzo Lombardo ${ }^{1}$, Antonio Pizzo ${ }^{2}$, Rossana Damiano ${ }^{1}$, Carmi Terzulli ${ }^{1}$, \\ and Giacomo Albert ${ }^{2}$ \\ 1 Department of Computer Science and CIRMA, University of Torino \\ 2 Department of Humanities and CIRMA, University of Torino \\ \{vincenzo.lombardo, antonio.pizzo, rossana.damiano, carmi.terzulli\}@unito.it
}

\begin{abstract}
This paper presents a visualization of stories that aligns the hierarchy of story units and the hierarchy of characters' intentions, respectively, with the story text, subdivided into chunks. The solution takes inspiration from the design introduced by the movie narrative charts, and presents an interactive tool.
\end{abstract}

Keywords: intelligent narrative technologies · interactive visualization - character intentions · story structure

\section{Introduction}

The visualization of stories and story-related facts has gained much importance in the recent literature and art. This is particularly true for stories with intricate plots that are not immediate to grasp (see, e.g., the visualization of two Nolan's films Memento $^{3}, 2000$, and Inception ${ }^{4}$, 2010). A source of inspiration for a number of approaches have been the Movie Narrative Charts by Munroe ${ }^{5}$ ), where the layout centers upon the notion of destiny of each character, depicted as a horizontal line, which travels from left to right and becomes adjacent to others when the characters interact in the story. This design layout has been automatized through algorithmic approaches in [5], [8] and later by [1], with increasing levels of legibility and performance. [3] introduce the motivations of character into the visualization: characters strive to achieve their goals and often end up conflicting with each other; the visualization aligns the sequence of the story incidents with the characters' intentions through a chart. The box-based design layout succeeds in fleshing out the story units (named "beats", scenes, sequences and acts in the literature, depending on their extension $[4,7]$ ) and the interpretation of characters' intentions (derived from AI models of agency, with short-, mid-, and long-term goals organized hierarchically [6, 2]), but misses the immediacy of line-based design and thus the representation of the continuity of characters' behavior. Also, interactivity is limited to the retrieval of textual box content.

\footnotetext{
${ }^{3}$ http://visual.ly/memento-scene-timeline

${ }^{4}$ http://visual.ly/inception-timeline-visualisation

${ }^{5}$ http://store.xkcd.com/collections/posters/products/ movie-narrative-charts-poster
} 
In this paper, we build on the character-based story-visualization design by introducing the hierarchical organization of the story incidents and the alignment with the characters' intentions. The result is an interactive tool that takes as input the hierarchical story structure and the hierarchical characters' intentions and visualizes the alignment between such hierarchies and the story text units: in particular, the hierarchies span some portion of the text and hierarchy elements are aligned with text chunk boundaries. The tool provides zoom and pan facilities, hide/show toggles, and text balloons for exploring the visualization and appraising the mapping of the story hierarchies over the portions of text. The design phase of the tool relied on the involvement of experts through qualitative methods of analysis, in particular a focus group conducted with experts.

Now we describe our design solution and the implementation of the visualization interface (Section 2). Section 3 proposes a case study on Shakespeare's Hamlet and the discussion about the results as well as some reflections on the lesson learnt. Conclusion ends the paper.

\section{Visualization design}

The design layout puts the sequence of story incidents, called timeline, in the middle of a chart representation (see Figure 1).

Above the timeline, we have the hierarchy of the incidents, organized into scenes of increasing large span (scenes, acts, play). Scenes are represented as grey arcs, spanning from their initial to their final incident; another colored arc, doubling the grey arc, marks the characters that are participating within that scene. A rectangle allows for the interactive inspection of the incident description in detail.

Below the timeline, separate tracks represent the individual characters, each in a different color. Each track features a header that allows for the inspection of the character details through a circle with the character's initials. Within the tracks, each intention is again represented as an arc; short-term to long-term intentions are visualized with different heights within the track size; smaller size intentions are included into the span of the larger size intentions. As mentioned above, the colors of the characters who participate into some incident structure are reported onto the arcs of the incident structure hierarchy, displayed above the timeline. When intentions are not accomplished in the timeline of incidents, they are visualized partially and with a barred rectangle.

The visualization is an interactive display that allows zooming into the representation, in order to visualize small portions of the timeline together with their accompanying structures, and pan from left to right and top to bottom, in order to explore the various areas of the visualization. In particular, zoom allows the user to focus on a given incident structure, with the colors on the structure arc to help her/him to figure out what characters participate into the incident; then, panning from top to bottom allows the user to discover what are the intentions of the individual characters in coincidence with the incident; passing the mouse over the rectangles that are associated with the arcs reveals 


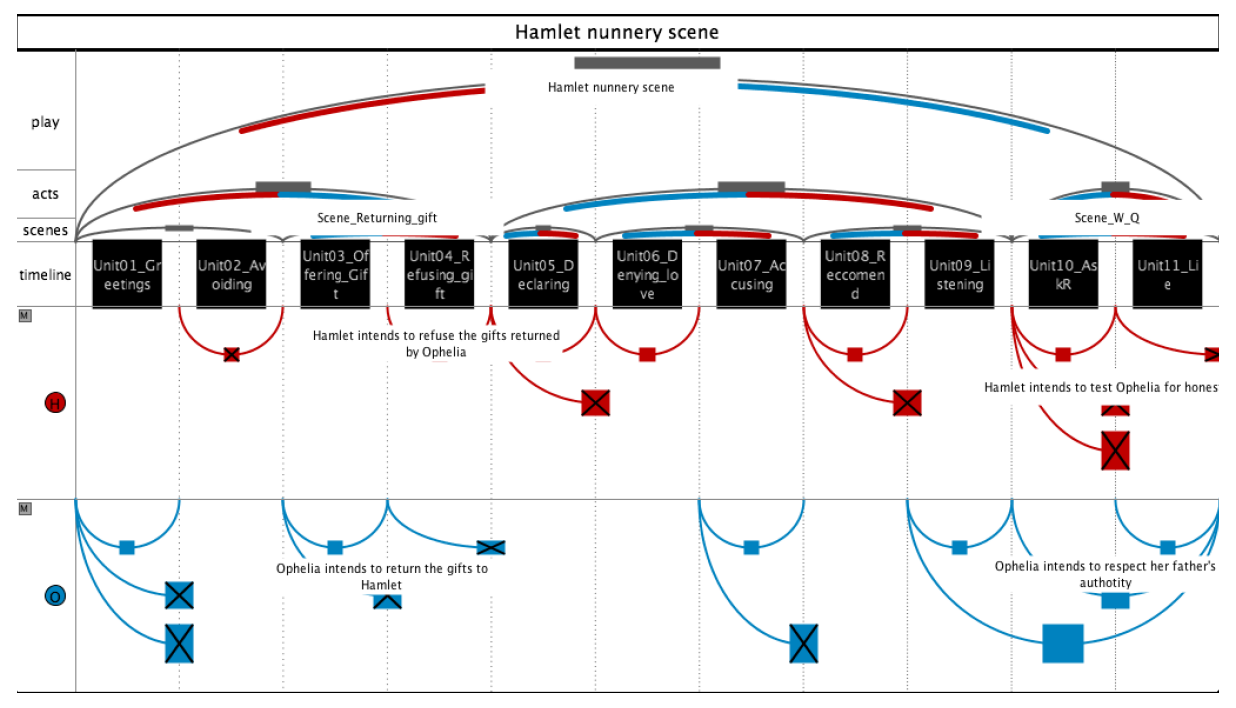

Fig. 1. Visualization of Hamlet nunnery scene. Through the tooltip facility of the interface we have highlighted two scenes with the intentions (in conflict) hinging on those scenes.

the detail of the intentions, while passing over an incident box reveals the details of the incident. Finally, we added a feature, taken from the audio editors, where tracks can be muted or played solo: we introduced a toggle to the track headers that excludes/includes some character track from the display, respectively.

The visualization tool takes as input an annotated table of events that includes the intentions of the characters and their eventual accomplishment. The tool is implemented in Processing ${ }^{6}$, after a pre-processing phase that extracts the individuals and relations from the tables provided by annotation.

\section{Case study}

As a case study, we have applied the visualization technique to a scene from Shakespeare's Hamlet, the "nunnery scene" (visualized in Figure 1). Notice the hierarchical representation of the intentions of the characters, with more complex intentions encompassing shorter, simpler intentions that only span one segment of the timeline. The number of plan failures spanning the same segment signal the high level of conflict that characterizes this part of the play. Failed intentions are represented by incomplete arcs marked by a crossed box. For example, Ophelia fails in returning the gifts to Hamlet at some point.

The succession of intentions displayed by a character's track represents the character's changes through planning and re--planning because of the conflicts

\footnotetext{
${ }^{6}$ http://processing.org/
} 
with other characters, thus stress the emotional charge of the drama. This is particular evident in the case of Ophelia (Figure 1): she has two main intentions (bottom of the figure) separated by a gap filled by one of Hamlet's intentions. This shows that Ophelia has to execute some sort of re-planning, given the failure of the first (bottom left), so to regain the lead of the scene with another overarching plan (bottom right). Moreover, all along the scene we see that there is a large number of failed intentions (rectangles barred with a cross); hence, the visualization reveals the inner nature of this scene with failed attempts on both sides to achieve their goals: on the one hand, Ophelia wants to discover the motivation for Hamlet's madness, on the other, Hamlet wants to send the "fair Ophelia" to the nunnery, but discovers that she is not honest at all.

\section{Conclusion}

This paper has presented an approach for the visualization of stories, based on the alignment between the incident sequence and the hierarchies of scenes and characters' intentions, respectively. The major novelty of the visualization is the introduction of the characters' tracks, that represent multiple hierarchies that span the sequence of story incidents.

Our approach is more analytic than existing ones with respect to addressing the basic constituents of a story; for example, some of the visualizations proposed elsewhere could be derived from our visualization. We are going to produce a large dataset of visualized dramas and to introduce the visualization tool systematically within drama analysis classes and to test its usefulness thoroughly.

\section{References}

1. Liu, S., Wu, Y., Wei, E., Liu, M., Liu, Y.: Storyflow: Tracking the evolution of stories. IEEE Transactions on Visualization and Computer Graphics 19(12), 24362445 (Dec 2013), http://dx.doi.org/10.1109/TVCG.2013.196

2. Lombardo, V., Battaglino, C., Pizzo, A., Damiano, R., Lieto, A.: Coupling conceptual modeling and rules for the annotation of dramatic media. Semantic Web Journal 6(5), 503-534 (2015)

3. Lombardo, V., Pizzo, A.: Ontology-based visualization of characters' intentions. In: International Conference on Interactive Digital Storytelling, pp. 176-187. Springer (2014)

4. McKee, R.: Story. Harper Collins, New York (1997)

5. Ogawa, M., Ma, K.L.: Software evolution storylines. In: Proceedings of the international symposium on Software visualization. pp. 35-42 (2010)

6. Rao, A., Georgeff, M.: BDI agents: From theory to practice. In: Proceedings of the First International Conference on Multi-Agent Systems (ICMAS'95) (1995)

7. Spencer, S.: The Playwright's Guidebook: An Insightful Primer on the Art of Dramatic Writing. Faber \& Faber (2002), http://books.google.it/books?id= nDrHmckSqi4C

8. Tanahashi, Y., Ma, K.L.: Design considerations for optimizing storyline visualizations. IEEE Transactions on Visualization and Computer Graphics 18(12), 2679 $2688(2012)$ 